PUPT - 1455, March 1994

\title{
Fermions Coupled to a Conformal Boundary: A Generalization of the Monopole-Fermion System
}

\author{
Ali Yegulalp* \\ Joseph Henry Laboratories \\ Princeton University \\ Princeton, NJ 08544
}

\begin{abstract}
We study a class of models in which $N$ flavors of massless fermions on the half line are coupled by an arbitrary orthogonal matrix to $N$ rotors living on the boundary. Integrating out the rotors, we find the exact partition function and Green's functions. We demonstrate that the coupling matrix must satisfy a certain rationality constraint, so there is an infinite, discrete set of possible coupling matrices. For one particular choice of the coupling matrix, this model reproduces the low-energy dynamics of fermions scattering from a magnetic monopole. A quick survey of the Green's functions shows that the S-matrix is nonunitary. This nonunitarity is present in previous results for the monopole-fermion system, although it appears not to have been noted. We indicate how unitarity may be restored by expanding the Fock space to include new states that are unavoidably introduced by the boundary interaction.
\end{abstract}

\footnotetext{
* yegulalp@puhep1.princeton.edu
} 


\section{Introduction}

We consider the action

$$
\begin{aligned}
A & =\int d t\left[\frac{1}{2} \sum_{i=1}^{N} \frac{\dot{\alpha}_{i}(t)^{2}}{I_{i}}+\sum_{i, j=1}^{N} \alpha_{i}(t) M_{i j}\left(: \psi_{j}^{\dagger}(0, t) \psi_{j}(0, t):-: \bar{\psi}_{j}^{\dagger}(0, t) \bar{\psi}_{j}(0, t):\right)\right. \\
& \left.+i \sum_{i=1}^{N} \int_{0}^{L} d x\left(\psi_{i}^{\dagger}(x, t)\left(\partial_{t}-\partial_{x}\right) \psi_{i}(x, t)+\bar{\psi}_{i}^{\dagger}(x, t)\left(\partial_{t}+\partial_{x}\right) \bar{\psi}_{i}(x, t)\right)\right]
\end{aligned}
$$

where $\psi_{i}$ and $\bar{\psi}_{i}$ are chiral components of Dirac fermion fields, $M_{i j}$ is an arbitrary real, orthogonal matrix, $\alpha_{i}$ are the rotor degrees of freedom, and the $I_{i}$ are constants. In writing the action, we have split the Dirac fermions into their two single-component chiral constituents, $\psi_{i}$ and $\bar{\psi}_{i}$, with the index $i$ labeling flavors.

The action describes $\mathrm{N}$ flavors of Dirac fermions living on the interval $0 \leq x \leq L$. There are no interactions except at $x=0$, where the fermion currents are linearly coupled to $\mathrm{N}$ rotor coordinates. The interaction at $x=0$ dynamically couples the left movers $\psi_{i}$ to the right movers $\bar{\psi}_{i}$ through the rotor coordinates. At $x=L$, we impose the simple reflecting boundary condition $\psi_{i}(L, t)=e^{2 \pi i \lambda_{i}} \bar{\psi}_{i}(L, t)$ so that the system will be closed and self-contained.

In general, the coupling at $x=0$ allows the fermions and the rotors to exchange energy, but we will be interested purely in the cases where energy is not exchanged: namely, when $I_{i} \rightarrow \infty$ or $I_{i} \rightarrow 0$. If we take all the $I_{i} \rightarrow \infty$, the rotors decouple and a simple reflecting boundary condition is imposed on the fermion currents at $x=0$. If we take some $I_{i} \rightarrow \infty$ and the others to zero, then things are much more interesting; we will find that we can integrate out the rotators, leaving behind a non-trivial, conformally invariant boundary condition on the fermions. The particular boundary condition obtained depends on which $I_{i} \rightarrow 0$ and the value of the matrix $M_{i j}$.

An interesting special case arises when we chose $M_{i j}$ and $I_{i}$ so that the action becomes essentially equivalent* to one first considered by Polchinski [1]. Polchinski's action captures the essential physics of charged fermions scattering from a magnetic monopole in four dimensions.

* Polchinksi uses right moving Weyl fermions on the full line, while we consider right and left moving Dirac fermions on the half line. One can convert the half-line Dirac theory into a full-line Weyl theory by defining $\bar{\psi}_{i}(-x, t)=\psi_{i}(x, t)$ for $x>0$, so that left movers at $x>0$ get reflected into right movers at $x<0$. 


\section{The Partition Function}

We use standard bosonization techniques [2] to rewrite the action in terms of the boson fields $\Phi_{i}$ :

$$
\begin{array}{r}
A=\int d t\left[\frac{1}{2} \sum_{i=1}^{N} \frac{\dot{\alpha}_{i}(t)^{2}}{I_{i}}+\sum_{i, j=1}^{N} \alpha_{i}(t) M_{i j} \dot{\Phi}_{j}(0, t)+\right. \\
\left.\frac{1}{8 \pi} \sum_{i=1}^{N} \int_{0}^{L} d x\left(\dot{\Phi}_{i}(x, t)^{2}-\Phi_{i}^{\prime}(x, t)^{2}\right)\right],
\end{array}
$$

where $\dot{\Phi}=\partial_{t} \Phi$ and $\Phi^{\prime}=\partial_{x} \Phi$. The bosons are compact, so $\Phi_{i} \equiv \Phi_{i}+2 \pi$. We fix $\Phi_{i}(L, t)=\theta_{i}$, where $\theta_{i}=\pi\left(1-2 \lambda_{i}\right)$, and allow $\Phi_{i}(0, t)$ to vary with the action. The classical equations of motion obtained by varying the action are

$$
\begin{array}{r}
\ddot{\alpha}_{i}(t)=I_{i} \sum_{j=1}^{N} M_{i j} \dot{\Phi}_{j}(0, t) \\
\Phi_{j}^{\prime}(0, t)-4 \pi \sum_{i=1}^{N} \dot{\alpha}_{i}(t) M_{i j}=0 \\
\Phi_{i}^{\prime \prime}(x, t)-\ddot{\Phi}_{i}(x, t)=0
\end{array}
$$

Eliminating the rotor coordinates $\alpha_{i}$ and changing over to the 'rotated' fields $\tilde{\Phi}_{i}(x, t)=$ $\sum_{j=1}^{N} M_{i j} \Phi_{j}(x, t)$, we get

$$
\begin{aligned}
\tilde{\Phi}_{i}^{\prime \prime}(x, t)-\ddot{\tilde{\Phi}}_{i}(x, t) & =0 \\
4 \pi I_{i} \tilde{\Phi}_{i}(0, t)+\tilde{\Phi}_{i}^{\prime}(0, t)-C_{i} & =0,
\end{aligned}
$$

where the $C_{i}$ are constants of integration.

At this point, let us consider the limit $I_{i} \rightarrow \infty$ for all $i$, which should reproduce the free fermion theory. The bosons are subject to the boundary conditions $\Phi_{i}(0, t)=$ $0, \Phi_{i}(L, t)=\theta_{i}$ and the compactness condition $\Phi_{i}(x, t) \equiv \Phi_{i}(x, t)+2 \pi$, which result in the following standard free partition function:

$$
Z=\prod_{n=1}^{\infty}\left(1-q^{n}\right)^{-N} \prod_{i=1}^{N}\left[\sum_{n=-\infty}^{\infty} q^{\frac{1}{2}\left(n+\frac{\theta_{i}}{2 \pi}\right)^{2}}\right],
$$

where $q=e^{-\pi \beta / L}$ and $\beta=$ inverse temperature. Note the presence of the factor $\sum_{n=-\infty}^{\infty} q^{\frac{1}{2}\left(n+\frac{\theta_{i}}{2 \pi}\right)^{2}}$, which is simply a sum over winding number. Without the compactness condition on $\Phi_{i}$, this factor would not be present. 
For the fermion system, we have the boundary conditions $\psi_{i}(0, t)=\bar{\psi}_{i}(0, t)$ and $\psi_{i}(L, t)=e^{2 \pi i \lambda_{i}} \bar{\psi}_{i}(L, t)$. This yields the partition function

$$
Z=\prod_{i=1}^{N} \prod_{n=-\infty}^{\infty}\left(1+q^{\left|n-\lambda_{i}\right|}\right)^{2}
$$

An application of Jacobi's triple product formula shows that the bosonic and fermionic partition functions are indeed equal up to a shift of zero-point energy, verifying our choice of boundary conditions for the bosonized action.

Now let us consider the general case $I_{i} \rightarrow \infty$ for $1 \leq i \leq a$ and $I_{i} \rightarrow 0$ for $a<i \leq N$. We find that

$$
\begin{array}{rr}
\tilde{\Phi}_{i}^{\prime \prime}(x, t)-\ddot{\tilde{\Phi}}_{i}(x, t)=0, & 1 \leq i \leq N \\
\tilde{\Phi}_{i}(0, t)=0, & 1 \leq i \leq a \\
\tilde{\Phi}_{i}^{\prime}(0, t)=0, & a<i \leq N \\
\tilde{\Phi}_{i}(L, t)=\sum_{j=1}^{N} M_{i j} \theta_{j}, & 1 \leq i \leq N \\
\tilde{\Phi}_{i}(x, t) \equiv \tilde{\Phi}_{i}(x, t)+2 \pi \sum_{j=1}^{N} M_{i j} n_{j}, & n_{j} \in \mathbb{Z}
\end{array}
$$

In principle, there should be a constant of integration in equation (2.60), but we have set it to zero because the rotor coordinate will carry an infinite amount of energy otherwise.

Since equations (2.6a) - (2.6e) describe a set of $N$ uncoupled free bosons, it seems that we should be able to compute the partition function as a product of $N$ independent partition functions. The fact that $M$ is an orthogonal matrix means that the canonical commutation relations are

$$
\left[\tilde{\Phi}_{i}(x, t), \dot{\tilde{\Phi}}_{j}(y, t)\right]=\left[\Phi_{i}(x, t), \dot{\Phi}_{j}(y, t)\right]=4 \pi i \delta_{i j} \delta(x-y)
$$

so there is no problem with quantizing the system in terms of the $\tilde{\Phi}_{i}$. The only catch is that we must take care in treating the winding modes. When the boundary condition at $x=0$ is $\tilde{\Phi}_{i}^{\prime}(0, t)=0$, it turns out that no winding mode exists, even though $\tilde{\Phi}_{i}(x, t)$ is compact. This lead us to the partition function

$$
Z=\prod_{n=1}^{\infty}\left(1-q^{n}\right)^{-a}\left(1-q^{\left(n-\frac{1}{2}\right)}\right)^{-(N-a)} \sum_{\left(\tilde{n}_{1}, \ldots \tilde{n}_{a}\right) \in \mathcal{Z}_{M}} q^{\frac{1}{2} \sum_{i=1}^{a}\left(\tilde{n}_{i}+\tilde{\theta}_{i}\right)^{2}}
$$


where

$$
\begin{aligned}
\tilde{\theta}_{i} & =\sum_{j=1}^{N} M_{i j} \frac{\theta_{j}}{2 \pi} \\
\mathcal{Z}_{M} & =\left\{\left(\tilde{n}_{1}, \ldots \tilde{n}_{a}\right) \mid \tilde{n}_{i}=\sum_{j=1}^{N} M_{i j} n_{j}, n_{j} \in \mathbb{Z}\right\} .
\end{aligned}
$$

The partition function almost looks like that of a free theory, except that the sum over winding modes is rather peculiar. Barring the trivial cases $a=0$ and $a=N$, the winding mode sum no longer factors into a product of independent winding mode sums. Instead, the winding modes of the different boson flavors are coupled together by the matrix $M$.

Furthermore, it seems that the winding number sum in the partition function does not make sense unless we require $M$ to satisfy a certain rationality property, the details of which will be explained in the next section. When $M$ satisfies the rationality property, the set $\mathcal{Z}_{M}$ is a discrete lattice, so points in $\mathcal{Z}_{M}$ are separated by finite gaps. When $M$ does not satisfy the rationality property, the set $\mathcal{Z}_{M}$ becomes dense on $\mathbb{R}^{a}$. Since we sum over the points of $\mathcal{Z}_{M}$ in the winding number sum, we find that the partition function diverges due to the infinitesimally close spacing of energy levels.

To flesh out the substance of the preceding remarks, we will work out a couple of simple examples: the general $N=2$ case and the magnetic monopole for arbitrary $N$.

3. $N=2$

Let us consider $N=2, a=1, \theta_{i}=0$, and write $M$ in the form

$$
M=\frac{1}{\sqrt{1+r^{2}}}\left(\begin{array}{cc}
1 & r \\
-r & 1
\end{array}\right) .
$$

This gives us

$$
\mathcal{Z}_{M}=\left\{\tilde{n} \mid \tilde{n}=\frac{n_{1}+r n_{2}}{\sqrt{1+r^{2}}} ; n_{1}, n_{2} \in \mathbb{Z}\right\} .
$$

Using some elementary number theory [3], we see that $\mathcal{Z}_{M}$ becomes dense on $\mathbb{R}$ unless $r$ is rational. For $N=2$, the constraint $r \in \mathbb{Q}$ is the rationality condition for $M$. Writing $r=p / l$ for $p, l \in \mathbb{Z}$ with $\operatorname{gcf}(p, l)=1$, we find that $\mathcal{Z}_{M}=\frac{1}{\sqrt{p^{2}+l^{2}}} \mathbb{Z}$, so the partition function is

$$
Z=\prod_{n=1}^{\infty}\left(1-q^{n}\right)^{-1}\left(1-q^{\left(n-\frac{1}{2}\right)}\right)^{-1} \sum_{m=-\infty}^{\infty} q^{\frac{m^{2}}{2\left(p^{2}+l^{2}\right)}}
$$


Using standard techniques [1], the partition function may be reexpressed in terms of $\tilde{q}=e^{\frac{4 \pi^{2}}{\ln (q)}}$ :

$$
Z=\sqrt{\frac{p^{2}+l^{2}}{2}} \tilde{q}^{-\frac{1}{12}} q^{\frac{1}{48}} \prod_{n=1}^{\infty}\left(1-\tilde{q}^{n}\right)^{-1}\left(1-\tilde{q}^{(2 n-1)}\right) \sum_{n=-\infty}^{\infty} \tilde{q}^{\frac{1}{2}\left(p^{2}+l^{2}\right) n^{2}}
$$

Now we can easily take the limit $L \rightarrow \infty$ :

$$
\ln (Z) \rightarrow \frac{1}{2} \ln \left(\frac{p^{2}+l^{2}}{2}\right)+\frac{\pi L}{3 \beta}+O\left(e^{-L / 2 \beta}\right)
$$

In addition to the standard piece in $\ln (Z)$ which scales linearly in the size of the system, we see that there is an $L$-independent term. If we associate the size-independent term with the boundary interaction at $x=0$, we find that the boundary contributes a temperature-

independent entropy $S_{b}=\frac{1}{2} \ln \left(\frac{p^{2}+l^{2}}{2}\right)$. Equivalently, this means that there are $g$ states associated with the boundary, where $g=e^{S_{b}}=\sqrt{\frac{p^{2}+l^{2}}{2}}$. The fact that $g$ need not be an integer may seem peculiar, but it is an unavoidable consequence of the way the winding modes for different bosons become linked at the boundary. The crucial point is that $g>1$, giving us a hint that a correct treatment of scattering may need to take into account some hidden degree of freedom on the boundary.

\section{The Magnetic Monopole}

Now we take $N \geq 2$, choose $a=N-1$ and $\theta_{i}=0$, and set

$$
M=\left(\begin{array}{ccccccc}
\frac{1}{\sqrt{2}} & -\frac{1}{\sqrt{2}} & 0 & 0 & \ldots & 0 & 0 \\
\frac{1}{\sqrt{6}} & \frac{1}{\sqrt{6}} & -\frac{2}{\sqrt{6}} & 0 & \ldots & 0 & 0 \\
\vdots & \vdots & \vdots & \vdots & \ddots & \vdots & \vdots \\
\frac{1}{\sqrt{N(N-1)}} & \frac{1}{\sqrt{N(N-1)}} & \frac{1}{\sqrt{N(N-1)}} & \frac{1}{\sqrt{N(N-1)}} & \cdots & \frac{1}{\sqrt{N(N-1)}} & -\frac{\left(N_{-1)}\right.}{\sqrt{N(N-1)}} \\
\frac{1}{\sqrt{N}} & \frac{1}{\sqrt{N}} & \frac{1}{\sqrt{N}} & \frac{1}{\sqrt{N}} & \cdots & \frac{1}{\sqrt{N}} & \frac{1}{\sqrt{N}}
\end{array}\right) .
$$

The last row of $M$ causes the sum of the fermion currents to be coupled to a rotor $\alpha_{N}$ which has $I \rightarrow 0$. The first $N-1$ rows of $M$ couple to rotors with $I \rightarrow \infty$, ensuring that differences of fermion currents obey reflecting boundary conditions at $x=0$. In Polchinski's version [1], there is just a single rotor, which correponds to our $\alpha_{N}$. The essence of the model is that we are changing only the boundary condition on the current which carries the total $U(1)$ charge. 
The only bit of work we need to perform is to find a convenient way to make the sum over $\mathcal{Z}_{M}$ explicit. If we think of the numbers $\tilde{n}_{i}$ as functions $\tilde{n}_{i}\left(n_{1}, n_{2}, \ldots, n_{N}\right)=$ $\sum_{j=1}^{N} M_{i j} n_{j}$, then we see that $\tilde{n}_{i}\left(n_{1}, n_{2}, \ldots, n_{N-1}, n_{N}+1\right)=\tilde{n}_{i}\left(n_{1}-1, n_{2}-1, \ldots, n_{N-1}-\right.$ $\left.1, n_{N}\right)$ for $1 \leq i \leq N-1$, so we can fix $n_{N}=0$ and just sum over $n_{1}, n_{2}, \ldots, n_{N-1}$. Note that we don't care about the value of $\tilde{n}_{N}$ since it does not appear in the winding mode sum. Furthermore, summing over $n_{1}, n_{2}, \ldots, n_{N-1}$ gives us each possible value of $\tilde{n}_{i}$ exactly once; this is true because the matrix obtained by deleting the last row and column of $M$ is a non-singular matrix. Finally, we note that $\sum_{i=1}^{N-1} \tilde{n}_{i}^{2}=\sum_{i=1}^{N} n_{i}^{2}-\frac{1}{N}\left(\sum_{i=1}^{N} n_{i}\right)^{2}$ Our desired partition function is

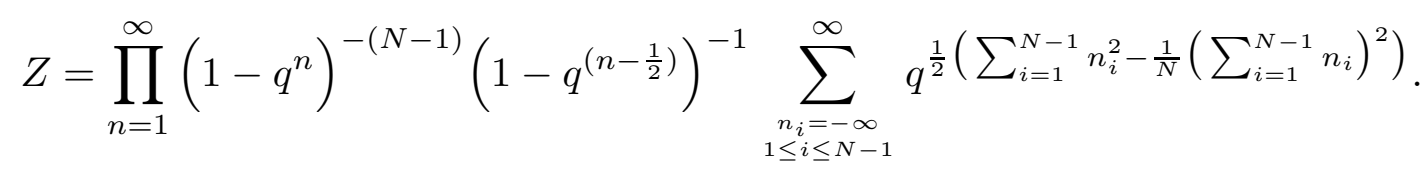

As in the $N=2$ case, we can express $Z$ in terms of $\tilde{q}=e^{\frac{4 \pi^{2}}{\ln (q)}}$ :

$$
Z=\frac{\tilde{q}^{-\frac{N}{24}} q^{\frac{N}{24}-\frac{1}{16}}}{\sqrt{2^{N} \operatorname{det}(A)}} \prod_{n=1}^{\infty}\left(1-\tilde{q}^{n}\right)^{-(N-1)}\left(1-\tilde{q}^{2 n-1}\right) \sum_{\substack{n_{i}=-\infty \\ 1 \leq i \leq N-1}}^{\infty} \tilde{q}^{\frac{1}{4} \sum_{i, j=1}^{N-1}\left(A^{-1}\right)_{i j} n_{i} n_{j}},
$$

where $A_{i j}=-\frac{1}{2 N}+\frac{1}{2} \delta_{i j}$. $\operatorname{det}(A)$ may be evaluated using a standard formula for circulants [5], yielding $\operatorname{det}(A)=2^{1-N} \frac{1}{N}$. Taking $L \rightarrow \infty$, we find the boundary degeneracy to be $g=\sqrt{\frac{N}{2}}$. Note that $g=1$ when $N=2$, so we should expect that there are no degrees of freedom on the boundary when $N=2$.

\section{Green's Functions}

Now we set $L=\infty$ and proceed to compute an arbitrary fermionic Green's function $\Gamma$ :

$$
\Gamma=<0\left|\bar{\psi}_{i_{1}}\left(\bar{z}_{1}\right) \ldots \bar{\psi}_{i_{p}}^{\dagger}\left(\bar{z}_{p}\right) \psi_{j_{1}}\left(w_{1}\right) \ldots \psi_{j_{q}}^{\dagger}\left(w_{q}\right)\right| 0>_{B},
$$

where we have switched to holomorphic coordinates $z=\tau+i x$ and imaginary time $\tau=i t$. To distinguish between the presence of $\psi$ and $\psi^{\dagger}$, we assign $F_{k}=1$ for $\bar{\psi}_{i_{k}}, F_{k}=-1$ for $\bar{\psi}_{i_{k}}^{\dagger}, G_{k}=1$ for $\psi_{j_{k}}$, and $G_{k}=-1$ for $\psi_{j_{k}}^{\dagger}$. The notation $<\ldots>_{B}$ indicates an expectation value in the presence of the boundary interaction at $x=0$, while $\langle\ldots\rangle$ will be used to indicate an expectation value for a free theory on the full line with no boundary interaction. 
As in the finite volume case, we bosonize the system according to the standard correspondence $\psi_{i}(z)=e^{i \Phi_{i}^{L}(z)}$ and $\bar{\psi}_{i}(\bar{z})=e^{-i \Phi_{i}^{R}(\bar{z}) *}$. Defining $\tilde{\Phi}_{i}(z, \bar{z})=\sum_{j=1}^{N} M_{i j} \Phi_{j}(z, \bar{z})$, we find that

$$
\begin{aligned}
\partial_{z} \partial_{\bar{z}} \tilde{\Phi}_{i}(z, \bar{z})=0 & & \text { for } \Im(z)>0 \\
\tilde{\Phi}_{i}(z, \bar{z})=0 & & \text { for } 1 \leq i \leq a, z=\bar{z} \\
\left(\partial_{z}-\partial_{\bar{z}}\right) \tilde{\Phi}_{i}(z, \bar{z})=0 & & \text { for } a<i \leq N, z=\bar{z} .
\end{aligned}
$$

We can decompose $\tilde{\Phi}_{i}(z, \bar{z})$ into left and right moving fields $\tilde{\Phi}_{i}^{L}(z)$ and $\tilde{\Phi}_{i}^{R}(\bar{z})$, but we must remember that the decomposition is not unique since $\tilde{\Phi}_{i}(z, \bar{z})=\tilde{\Phi}_{i}^{L}(z)+\tilde{\Phi}_{i}^{R}(\bar{z})=$ $\left(\tilde{\Phi}_{i}^{L}(z)+C\right)+\left(\tilde{\Phi}_{i}^{R}(\bar{z})-C\right)$. Accordingly, let us define constants $\tilde{\omega}_{i}$ such that $<\tilde{\Phi}_{i}^{L}(z)>=$ $-<\tilde{\Phi}_{i}^{R}(\bar{z})>=\tilde{\omega}_{i}$. For convenience, define $\tilde{\phi}_{i}^{L}(z)=\tilde{\Phi}_{i}^{L}(z)-\tilde{\omega}_{i}$ and $\tilde{\phi}_{i}^{R}(\bar{z})=\tilde{\Phi}_{i}^{R}(\bar{z})+\tilde{\omega}_{i}$. Using equation (5.2), we can solve for the left movers in terms of the right movers:

$$
\begin{aligned}
\phi_{i}^{L}(z) & =\sum_{k=1}^{N} S_{i k} \phi_{k}^{R}(z) \\
S_{i k} & =-\sum_{j=1}^{N} \sigma_{j} M_{j i} M_{j k} \\
\sigma_{i} & =\left\{\begin{array}{ll}
1, & 1 \leq i \leq a \\
-1, & a<i \leq N
\end{array},\right.
\end{aligned}
$$

where $\phi_{i}=\sum_{j=1}^{N} M_{j i} \tilde{\phi}_{j}$.

The matrix $S$ is manifestly symmetric, and a short calculation shows that it is also orthogonal. Furthermore, any matrix which is real, symmetric, and orthogonal may be written in the form $S_{i k}=-\sum_{j=1}^{N} \sigma_{j} M_{j i} M_{j k}$ with $\sigma_{j}^{2}=1$ and $M$ real and orthogonal. From this, we see that the boundary interaction at $x=0$ merely 'rotates' the left movers with respect to the right movers, and we may choose the boundary interaction so that any particular symmetric and orthogonal matrix $S$ carries out the rotation.

In bosonized form, equation (5.1) becomes

$$
\Gamma=<0\left|e^{-i F_{1} \Phi_{i_{1}}^{R}\left(\bar{z}_{1}\right)} \ldots e^{-i F_{p} \Phi_{i_{p}}^{R}\left(\bar{z}_{p}\right)} e^{i G_{1} \Phi_{j_{1}}^{L}\left(w_{1}\right)} \ldots e^{i G_{q} \Phi_{j_{q}}^{L}\left(w_{q}\right)}\right| 0>_{B}
$$

* To avoid cumbersome anticommuting factors normally appearing in multi-flavor bosonization, we simply assume that $\Gamma$ is written with the fields in the canonical ordering $i_{1} \leq i_{2} \leq \ldots i_{p}$, $j_{1} \leq j_{2} \leq \ldots j_{q}$. 
Using equation (5.3) to write all the $\Phi_{i}^{L}(z)$ in terms of the $\Phi_{i}^{R}(z)$, we find that $\Gamma$ is simply the expectation value of a product of right moving chiral vertex operators. Once $\Gamma$ is expressed in terms of just right moving fields, we can forget about the presence of the boundary interaction and compute as we would in a free theory. Use standard techniques for vertex operators [4], we get

$$
\begin{aligned}
\Gamma= & e^{i \sum_{r=1}^{N} \omega_{r}\left(f_{r}+g_{r}\right)} \prod_{r=1}^{N} \delta_{f_{r}, \sum_{r^{\prime}=1}^{N} S_{r r^{\prime}} g_{r^{\prime}}} \prod_{\substack{\alpha, \beta=1 \\
\alpha<\beta}}^{p}\left(\bar{z}_{\alpha}-\bar{z}_{\beta}\right)^{F_{\alpha} F_{\beta} \delta_{i_{\alpha} i_{\beta}}} \prod_{\substack{\lambda, \nu=1 \\
\lambda<\nu}}^{q}\left(w_{\lambda}-w_{\nu}\right)^{G_{\lambda} G_{\nu} \delta_{j_{\lambda} j_{\nu}}} \\
& \prod_{\alpha=1}^{p} \prod_{\lambda=1}^{q}\left(\bar{z}_{\alpha}-w_{\lambda}\right)^{-F_{\alpha} G_{\lambda} S_{i_{\alpha} j_{\lambda}}}
\end{aligned}
$$

where $f_{r}=\sum_{\alpha=1}^{p} F_{\alpha} \delta_{i_{\alpha} r}$ and $g_{r}=\sum_{\lambda=1}^{q} G_{\lambda} \delta_{j_{\lambda} r}$. Note that $f_{r}$ and $g_{r}$ are simply the total amount of each flavor present in the ingoing and outgoing states, respectively.

For the magnetic monopole, $S_{i j}=-\delta_{i j}+\frac{2}{N}$, giving us

$$
\begin{aligned}
\Gamma_{\text {monopole }}=e^{i \kappa \omega} & \prod_{r=1}^{N} \delta_{f_{r}+g_{r}, \kappa} \prod_{\substack{\alpha, \beta=1 \\
\alpha<\beta}}^{p}\left(\bar{z}_{\alpha}-\bar{z}_{\beta}\right)^{F_{\alpha} F_{\beta} \delta_{i_{\alpha} i_{\beta}}} \prod_{\substack{\lambda, \nu=1 \\
\lambda<\nu}}^{q}\left(w_{\lambda}-w_{\nu}\right)^{G_{\lambda} G_{\nu} \delta_{j_{\lambda} j_{\nu}}} \\
& \prod_{\alpha=1}^{p} \prod_{\lambda=1}^{q}\left(\bar{z}_{\alpha}-w_{\lambda}\right)^{F_{\alpha} G_{\lambda}\left(\delta_{i_{\alpha} j_{\lambda}}-\frac{2}{N}\right)}
\end{aligned}
$$

where $\kappa=\frac{2}{N} \sum_{r=1}^{N} g_{r}$ and $\omega=\sum_{r=1}^{N} \omega_{r}$. Our results for the monopole Green's functions agree with the original calculation by Polchinski [1], in which he evaluated the path integral by integrating out the fermions. The parameter $\omega$ in our result corresponds to the vacuum instanton angle $\theta$ in Polchinski's result. The path integral calculation gives results which are integrated over all values of $\theta$, from which Polchinski extracted the $\theta$-vacuum Green's functions by cluster decomposition arguments. In our bosonized solution, we automatically obtain the Green's functions in a fixed $\theta$-vacuum sector.

\section{A Unitarity Paradox}

Now we would like to point out that the Green's functions of equation (5.6) produce an apparently nonunitary S-matrix for $N>2^{*}$. Let us consider Green's functions where

\footnotetext{
* The unitarity problem is not particular to our treatment of the monopole-fermion system;
} the results obtained in [1] have exactly the same problem, although it seems not to have been commented on until now. 
we have some fixed combination of left moving operators and any number of right moving operators. The idea here is that we fix an ingoing state created by left moving operators, allow scattering to take place, and then look at the overlap with outgoing states made of arbitrary combinations of right movers. Since we have fixed the left movers, the integer parameters $G_{\lambda}$ and $g_{r}$ are fixed, while $F_{\alpha}$ and $f_{r}$ are free to vary. Since $\kappa=\frac{2}{N} \sum_{r=1}^{N} g_{r}$, we see that $\kappa$ is fixed, but not necessarily integral for $N>2$. Looking at equation (5.6), we see that the Green's function will vanish unless $f_{r}+g_{r}=\kappa$ for all $r$, leading us to the following conclusions: for $N=2$, every choice of ingoing state has overlap with some outgoing states, and there is no problem with unitarity. In fact, the interaction at $N=2$ simply swaps the two flavors upon reflection from the boundary. For $N>2$, we find that any ingoing state with a non-integer $\kappa$ (i.e., a state whose total charge is not a multiple of $N / 2$ ) has zero overlap with all possible outgoing states, violating probability conservation and unitarity.

As a first step toward resolving the unitarity problem, let us think about the S-matrix in terms of bosons. The advantage of the bosonized form is that we can think of the scattering directly in terms of operators: by virtue of equation (5.3), we can think of the boundary S matrix as an operator which maps the chiral, left moving 'in' state operators into the chiral, right moving 'out' state operators. Explicitly, we have $\hat{S} \phi_{i}^{L}(z) \hat{S}^{-1}=$ $\sum_{j=1}^{N} S_{i j} \phi_{j}^{R}(z)$, where $\hat{S}$ is the Hilbert space S-matrix operator and we regard $\phi_{i}^{L}(z)$ and $\phi_{i}^{R}(z)$ as operators in a free theory without a boundary interaction.

Formally, it is easy to show that $\hat{S}$ acts on the bosonic Fock space in a unitary way. Let $\mid i n>$ and $\mid i n^{\prime}>$ denote two arbitrary ingoing states formed by acting on the vacuum $\mid 0>$ with arbitrary combinations of operators $\phi_{i}^{L}(z)$. To demonstrate unitarity, we need to show that $<i n^{\prime}\left|\hat{S}^{\dagger} \hat{S}\right| i n>=<i n^{\prime} \mid i n>$. If we let $\hat{S}$ and $\hat{S}^{\dagger}$ act on the left moving fields in $\mid i n>$ and $<i n^{\prime} \mid$, the left movers turn into right movers multiplied by factors of $S_{i j}$. Since we are now dealing with free field theory, the inner product may be evaluated in terms of two-point functions by Wick's theorem. The orthogonality of $S_{i j}$ implies that $<0\left|\phi_{i}^{L}(z) \hat{S}^{\dagger} \hat{S} \phi_{j}^{L}(w)\right| 0>=\sum_{k, l=1}^{N} S_{i k} S_{j l}<0\left|\phi_{k}^{R}(z) \phi_{l}^{R}(w)\right| 0>=<0\left|\phi_{i}^{L}(z) \phi_{j}^{L}(w)\right| 0>$, which finishes the proof that $\hat{S}$ is unitary.

Now let us consider what happens when $\hat{S}$ acts on fermionic states. Setting $N=4$ for simplicity, we find that

$$
\begin{aligned}
& \hat{S} \psi_{1}(z) \hat{S}^{-1}=\hat{S} e^{i \Phi_{1}^{L}(z)} \hat{S}^{-1}=e^{-\frac{i}{2} \Phi_{1}^{R}(z)} e^{\frac{i}{2} \Phi_{2}^{R}(z)} e^{\frac{i}{2} \Phi_{3}^{R}(z)} e^{\frac{i}{2} \Phi_{4}^{R}(z)} \\
& \hat{S} \psi_{2}(z) \hat{S}^{-1}=\hat{S} e^{i \Phi_{2}^{L}(z)} \hat{S}^{-1}=e^{\frac{i}{2} \Phi_{1}^{R}(z)} e^{-\frac{i}{2} \Phi_{2}^{R}(z)} e^{\frac{i}{2} \Phi_{3}^{R}(z)} e^{\frac{i}{2} \Phi_{4}^{R}(z)}
\end{aligned}
$$


with similar expressions for the other flavors. Every ingoing fermion operator scatters into a product of vertex operators carrying half-integer charges of each flavor. Since the expectation value of a product of vertex operators vanishes unless there is a net charge of zero for each flavor, we see that ingoing states with an odd number of fermions have no overlap with any outgoing states made of fermions. This suggests a simple solution to our unitarity problem: we should expand our Fock space and allow outgoing states to contain vertex operators carrying half-integer charges. The new operators are in fact nothing but spin operators for our Dirac fermions. The complete Fock space will be generated from fermion operators and spin operators, and the boundary interaction will couple the two kinds of operators together. We can also think of the Fock space as being built from fermion operators acting on two kinds of vacuum states: one is the ordinary Raymond vacuum, and the other is the Neveu-Schwarz vacuum created by a spin operator acting on the Raymond vacuum. When an even number of fermions scatter from the boundary, we remain in the same vacuum. When an odd number of fermions scatter from the boundary, the outgoing states emerge in the opposite vacuum from the ingoing states. In some sense, we can think of the boundary as having a two-fold degeneracy corresponding to the two possible vacua. We plan to present a more detailed exposition of these ideas in a forthcoming work.

\section{Conclusions}

In this letter we have generalized the monopole-fermion system to a class of conformal systems with conformal boundary interaction parameterized by an orthogonal matrix. Abelian bosonization allows completely explicit computation of the partition function and Green's functions, which agree with previous results obtained for the monopole-fermion system. We point out that the scattering appears to be nonunitary when $N>2$, and suggest how this may be corrected by recognizing the presence of additional states of the

boundary. The partition function tells us that the boundary degeneracy is $g=\sqrt{\frac{N}{2}}$, so extra states for the boundary are required precisely when $g>1$.

\section{Acknowledgements}

We would like to thank C. G Callan, A. W. W. Ludwig, I. Klebanov, and J. Maldacena for useful discussions and advice. This work was supported in part by DOE grant DEFG02-90ER40542. 


\section{References}

[1] J. Polchinski, Nucl. Phys. B242 (1984) 345.

[2] S. Coleman, Phys. Rev. D 11, 2088 (1975); S. Mandelstam, Phys. Rev. D11, 3026 (1975).

[3] I. Niven, H. S. Zuckerman, and H. L. Montgomery, An Introduction to the Theory of Numbers, 5th ed. (John Wiley \& Sons, Inc., 1991).

[4] M. B. Green, J. H. Schwarz, and E. Witten, Superstring Theory (Cambridge University Press, 1988).

[5] I. S. Gradshteyn and I. M. Ryzhik, Table of Integrals, Series, and Products, Alan Jeffrey ed. (Academic Press, Inc., 1980). 